\title{
Near-Infrared photometry of the LMC cluster Reticulum
}

\author{
M. Dall'Ora ${ }^{1,2}$, G. Bono ${ }^{2}$, J. Storm ${ }^{3}$, V. Ripepi ${ }^{4}$, V. Testa ${ }^{2}$, \\ G. Andreuzzi ${ }^{2}$, R. Buonanno ${ }^{1,2}$, F. Caputo ${ }^{2}$, V. Castellani ${ }^{2,5}$, \\ C.E. Corsi ${ }^{2}$, S. Degl'Innocenti ${ }^{6}$, G. Marconi ${ }^{2,7}$, M. Marconi $^{4}$, \\ M. Monelli ${ }^{1,2}$
}

${ }^{1}$ Dipartimento di Fisica, Università di Roma Tor Vergata, Via della Ricerca Scientifica, 1, 00133 Rome, Italy

${ }^{2}$ INAF-OAR, via Frascati 33, 00040 Monteporzio Catone, Italy

${ }^{3}$ Astrophysikalisches Institut Potsdam, An der Sternwarte 16, D-14482 Potsdam, Germany

${ }^{4}$ INAF, Osservatorio Astronomico di Capodimonte, via Moiariello 16 , 80131 Napoli, Italy

${ }^{5}$ INFN, Sezione di Pisa, via Livornese 582/A, S. Piero a Grado, 56010, Pisa, Italy

${ }^{6}$ Dipartimento di Fisica, Università di Pisa, piazza Torricelli 2, 56126 Pisa, Italy

${ }^{7}$ European Southern Observatory, Alonso de Cordova 3107, Casilla 19001, Vitacura, Santiago 19, Chile

\begin{abstract}
We present $U, B, V, I$ (SUSI@NTT) and $J, K_{s}$ (SOFI@NTT) photometry of the Large Magellanic Cloud (LMC) cluster Reticulum. The observing strategy and data reduction (DAOPHOTII/ALLFRAME) allowed us to reach an accuracy of the order of $0.01-0.03 \mathrm{mag}$ in all the bands at limiting magnitudes typical of RR Lyrae stars. Reticulum hosts a sizable sample of RR Lyrae stars (32), and we supply an accurate distance estimate using the RR Lyrae $K$-band Period-Luminosity-Metallicity $\left(P L Z_{K}\right)$ relation. This method presents several advantages when compared with the $M_{V}$ vs $[\mathrm{Fe} / \mathrm{H}]$ relation, since it is only marginally affected by off-ZAHB evolutionary effects and reddening corrections.
\end{abstract}

\section{Introduction}

RR Lyrae stars are good distance indicators for low-mass stellar populations, since they have approximately the same visual magnitude, are bright enough to be detected at moderately large distances (within the Local Group) and are easy to recognize from their light curves. Typically, their visual magnitude is calibrated as a linear function of the metallicity. Although RR Lyrae stars have been the crossroad of several theoretical and empirical investigations, both the zero point and the slope are still poorly constrained (see, e.g., Bono 2003, and 
references therein). Moreover, visual magnitudes are also dependent on the evolutionary status, and this information is not known a priori, even for the cluster RR Lyrae stars. Longmore, Fernley \& Jameson (1986) and Longmore et al. (1990) found that in the $K$-band RR Lyrae follow a well-defined empirical period-luminosity relation. Moreover, $K$ magnitudes are about a factor of ten less affected by reddening. On the basis of an extensive grid of non-linear, timedependent, convective models (Bono \& Stellingwerf 1994), Bono et al. (2001, 2003) derived a new $P L Z_{k}$ relation, furthermore demonstrating that $K$-band magnitudes show only a mild dependence on the luminosity, linked to their evolutionary status. Theory was tested on RR Lyrae itself, and Bono et al. (2002) obtained a "pulsational parallax" of $3.858 \pm 0.131$ mas, in good agreement with the trigonometric parallax $3.82 \pm 0.20$ mas obtained by Benedict et al. (2002) on HST data, but with a smaller formal error.

In this work we mainly present $K_{s}$-band observations of RR Lyrae stars in the LMC cluster Reticulum. We supply new distance moduli to Reticulum and to LMC by means of the $P L Z_{k}$ relation. Details on the infrared dataset can be found in Dall'Ora et al. (2004), while the optical dataset will be extensively discussed in a forthcoming paper (Ripepi et al., in preparation).

\section{Observations and data reduction}

We collected $46 J$ and $171 K_{s}$ images with SOFI@NTT with the large field setup $\left(4.5^{\prime} \times 4.5^{\prime}\right)$ in three different runs, from 1999 December to 2002 February. We secured approximately a dozen phase points both in $J$ and in $K_{s}$. Data were initially reduced with IRAF facilities, following the procedures outlined in the SOFI manual (Lidman et al. 2002). Photometry was performed by means of DAOPHOTII/ALLSTAR and ALLFRAME packages (Stetson 1987, 1994). Photometric zero points were calculated on the Persson et al. (1998) 9109 standard star. Optical observations $(U, B, V, I)$ were obtained with SUSI1/2@NTT during a period of about six years (Fig. 1). Collected phase points in the optical bands range approximately from 30 in the $I$-band to 130 in the $V$-band. We recovered 30 of the $32 \mathrm{RR}$ Lyrae stars known (Walker 1992). Intensity-averaged $K$-band mean magnitudes for the RR Lyrae stars were obtained by fitting observed phase points with the template $K$-band light curves provided by Jones et al. (1996), as shown in Fig. 1.

\section{Distance modulus and final remarks}

In Fig. 2 we show the $\log P-\langle K\rangle$ plot of RR Lyrae stars in Reticulum. First overtone pulsators (empty circles) have been "fundamentalized", i.e. we added 0.127 to their $\log P$. In the following we put $K=K_{s}$, since the difference is negligible for our goals, as reported in the SOFI user manuals (Lidman et al. 2002 ). For the estimate of the distance modulus, we used the relation

$$
M_{K}=-0.770-2.101 \log P+0.231[\mathrm{Fe} / \mathrm{H}]
$$

derived by Bono et al. (2003). Note that linear regression of data plotted in Fig. 2 gives a slope of $-2.26 \pm 0.14$. By adopting $[\mathrm{Fe} / \mathrm{H}]=-1.71 \pm 0.01$ (Suntzeff 

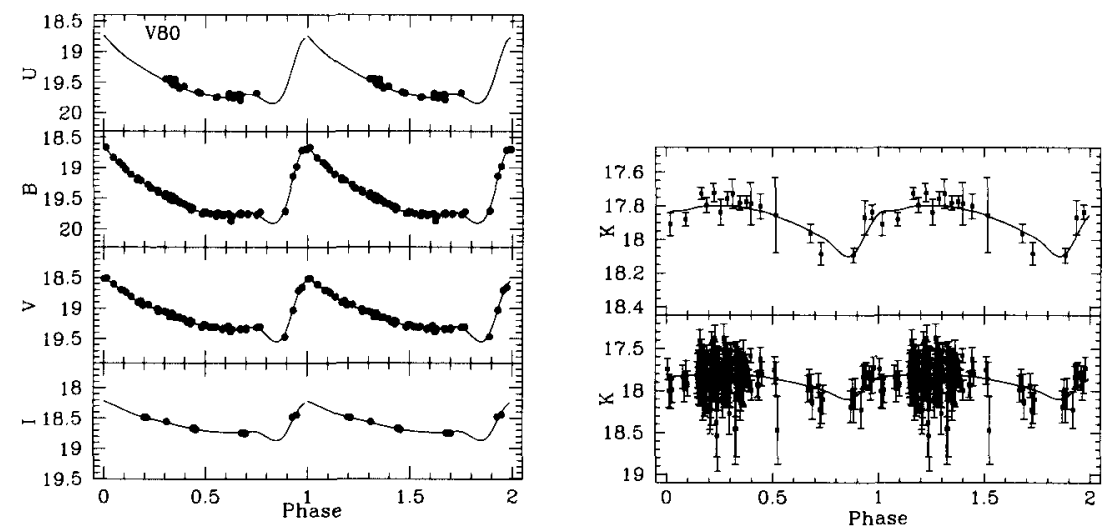

Figure 1. Left panel: optical light curves for the variable V80. Data points were fitted with a spline. When the number of observations was not sufficient to fit a spline, a scaled spline from $B$ and $V$ bands observations was used respectively for the $U$ and the $I$ bands. Right Panel: $K$-band observations (bottom) were fitted with the template by Jones et al. (1996). Observations were also averaged per phase bin (top), with a bin size of 0.03 phase.

et al. 1992), we found $(m-M)_{K}=18.534 \pm 0.005$ (intrinsic mean error). Using $E(B-V)=0.03 \pm 0.02$ ) (Walker 1992) and $A_{K}=0.114 A_{V}$ (Cardelli, Mathis, \& Clayton 1989), we obtain $A_{K}=0.01$ and therefore the true distance modulus to Reticulum is $(m-M)_{0}=18.524 \pm 0.005$. Assuming a difference in the distance between Reticulum and the center of LMC of $\delta \mu \approx 0.08$ (Walker 1992), and taking in account an uncertainty on the metallicity of $\approx 0.25 \mathrm{dex}$, that translates in an uncertainty of $0.06 \mathrm{mag}$, we obtain a distance to LMC of $\mu=18.444 \pm 0.065$. This is in good agreement with recent determinations available in the literature (Cacciari \& Clementini 2003; Alves 2003).

The present data support the evidence that the $P L Z_{K}$ relation is characterized by an intrinsic accuracy better than $0.1 \mathrm{mag}$. This means that $P L Z_{K}$ relation can supply a new distance scale to galactic globular clusters, thus substantially improving their absolute age estimates. Moreover, owing to the marginal dependence on the reddening, this relation could be also adopted to derive an accurate 3D structure of the Magellanic Clouds, as well as of the Galactic Bulge.

\section{References}

Alves, D.R. 2003, astro-ph/0310673

Benedict, G.F., et al. 2002, AJ, 123, 473

Bono, G. 2003, in Stellar Candles, eds W. Gieren \& D. Alloin, Lecture Notes in Physics (Berlin: Springer/Verlag), 85

Bono, G., Caputo, F., Castellani, V., Marconi, M., Storm, J. 2001, MNRAS, 326,1183 


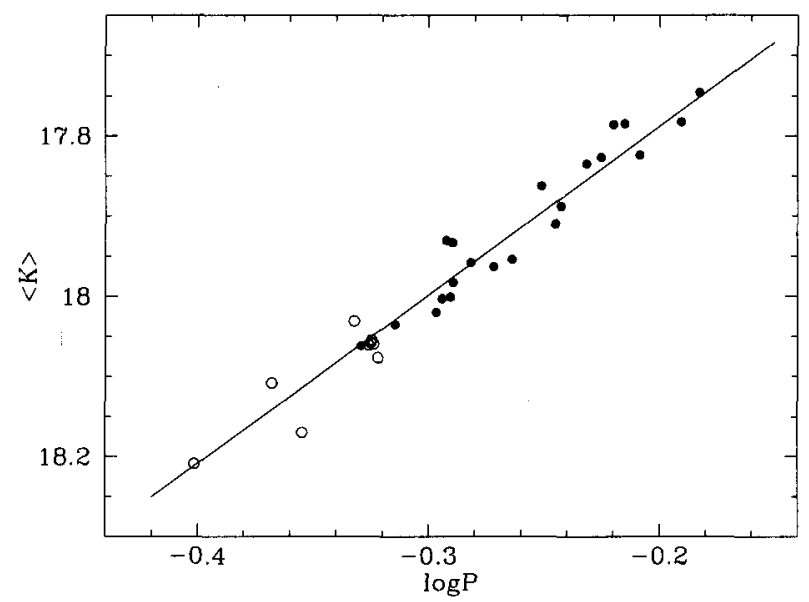

Figure 2. Intensity averaged $K$ mean magnitude as a function of $\log P$ for the RR Lyrae stars in Reticulum. The straight line represents Eq. 1 shifted to our best-fit distance.

Bono, G., Caputo, F., Castellani, V., Marconi, M., Storm, J. 2002, MNRAS, 332,78

Bono, G, Caputo, F., Castellani, V., Marconi, M., Storm, J., Degl'Innocenti, S. 2003, MNRAS, 344, 1097

Bono, G., Stellingwerf, R.F. 1994, ApJS, 93, 233

Cacciari, C., Clementini, G. 2003, in Stellar Candles, eds W. Gieren \& D. Alloin, Lecture Notes in Physics (Berlin: Springer/Verlag), 105

Cardelli, J.A., Clayton, G.C., Mathis, J.S. 1989, ApJ, 345, 245

Dall'Ora, M., et al. 2004, ApJL, submitted

Jones, R.V., Carney, B.W., Fulbright, J.P. 1996, PASP, 108, 877

Lidman C., Cuby J., Vanzi, L., Billeres, M., Hainaut O., Pompei, E. 2002, UserDoc. No. LSO-MAN-ESO-40100-0004, issue 1.4

Longmore, A.J., Dixon, R., Skillen, I., Jameson, R.F., Fernley, J.A. 1990, MNRAS, 247, 684

Longmore, A.J., Fernley, J.A., Jameson, R.F. 1986, MNRAS, 220, 279

Persson, S.E., Murphy, D.C., Krzeminski, W., Roth, M., Rieke, M.J. 1998, AJ, 116,2475

Stetson, P.B. 1987, PASP, 99, 191

Stetson, P.B. 1994, PASP, 106, 250

Suntzeff, N.B., Schommer, R.A., Olszewski, E.W., Walker, A.R. 1992, AJ, 104, 1743

Walker, A.R. 1992, AJ, 103, 1166 